\title{
Routine ganglionic plexi ablation during Maze procedure improves hospital and early follow-up results of mitral surgery
}

Francesco Onorati, MD, ${ }^{a}$ Antonio Curcio, MD, ${ }^{\mathrm{b}}$ Giuseppe Santarpino, MD, ${ }^{\text {a }}$ Daniele Torella, MD, ${ }^{\mathrm{b}}$ Pasquale Mastroroberto, MD, ${ }^{a}$ Luigi Tucci, MD, ${ }^{\mathrm{c}}$ Ciro Indolfi, MD, FESC, ${ }^{\text {, }}$ and Attilio Renzulli, MD, PhD, FECTS ${ }^{a}$

From the Cardiac Surgery Unit, ${ }^{\text {a }}$ Cardiology Unit, ${ }^{\mathrm{b}}$ Magna Graecia University of Catanzaro, Catanzaro, Italy; and Pathology Unit, ${ }^{c}$ Ospedale Pugliese-Ciaccio, Catanzaro, Italy.

Received for publication Dec 4, 2007; revisions received Feb 19, 2008; accepted for publication March 18, 2008.

Address for reprints: Francesco Onorati, MD, Viale dei Pini, 28, 80131 Napoli (E-mail: frankono@libero.it).

J Thorac Cardiovasc Surg 2008;136:408-18 $0022-5223 / \$ 34.00$

Copyright $(0) 2008$ by The American Association for Thoracic Surgery

doi:10.1016/j.jtcvs.2008.03.022
Objective: Ganglionic plexi are claimed to be potentially responsible for atrial fibrillation. We evaluated whether ganglionic plexi isolation improves the results of the Maze procedure during mitral valve surgery.

Methods: A total of 75 patients with atrial fibrillation underwent radiofrequency ablation during mitral valve surgery without (group A) or with (group B) ganglionic plexi ablation with bipolar radiofrequency plus fat pad resection along the Waterston groove, left pulmonary veins, and Marshall's ligament. Ganglionic plexi were intraoperatively mapped, and fat pad specimens were sectioned and analyzed. Hospital and follow-up results were recorded. Amiodarone was discontinued at the sixth month.

Results: Active ganglionic plexi were mainly located in the upper parts of fat pads. Active specimens demonstrated more ganglionic plexi than inactive specimens ( $P$ $\leq .015$ at different levels) but did not correlate with atrial fibrillation recurrence $(P=$ not significant). Atrial fibrillation was higher in group A at aortic declamping $(P=.03)$ and discharge $(P=.03)$. Early events were comparable $(P=.565)$. At $16.7 \pm 0.95$ (standard error) months, the cumulative freedom from atrial fibrillation, atrial flutter, and atrial tachycardia with antiarrhythmic therapy was $63.2 \% \pm 7.3 \%$ and proved higher in group B $(83.9 \% \pm 7.9 \%$ vs group A $52.8 \% \pm 8.7 \% ; P=$ $.035)$. However, after the sixth month, at $12.8 \pm 0.80$ months, freedom from atrial fibrillation, atrial flutter, and atrial tachycardia without antiarrhythmic therapy was $72.5 \% \pm 7.7 \%$ and proved higher in group B $(92.9 \% \pm 6.9 \%$ vs $62.5 \% \pm 9.4 \% ; P$ $=.023)$. A higher proportion of patients in group $\mathrm{B}$ showed normalized $\mathrm{E} / \mathrm{A}$ ratio $(61.3 \%$ vs group A $36.4 \% ; P=.029)$. No differences were detected in follow-up freedom from congestive heart failure (group A: $83.4 \% \pm 7.0 \%$ vs group B: $93.5 \% \pm$ $4.4 \% ; P=.978$ ) and hospital readmission (group A: $84.2 \% \pm 5.9 \%$ vs group B: $92.6 \% \pm 5.1 \% ; P=.376)$.

Conclusion: Ganglionic plexi isolation can improve hospital and follow-up results during mitral valve surgery and possibly ameliorate echocardiographic recovery of atrial function during follow-up.

$\mathrm{S}$ ince Cox proved that atrial fibrillation (AF) could be treated by means of surgery, ${ }^{1}$ a growing number of modified "mini-Maze" procedures have been proposed to overcome the problems of complexity and prolonged operating time of the Cox-Maze III technique., ${ }^{2,3}$ Along with the demonstration by Haissaguerre and colleagues ${ }^{4}$ of the key role of ectopic triggers localized in the pulmonary veins, recent literature data also have shown a role of epicardial ganglionic plexi (GP), which are primarily found in the epicardial fat tissue of the upper half part of the heart, in determining or inducing AF. ${ }^{5,6}$ Accordingly, some articles from electrophysiologic groups have addressed the safety, feasibility, and efficacy of sinus rhythm (SR) recovery after GP catheter ablation in paroxysmal AF. ${ }^{7,8}$ Furthermore, it was recently confirmed with electrophysiologic mapping that active GP are clustered around the interatrial groove and the ligament of Marshall, and that cardiac response to GP stimulation 


$$
\begin{aligned}
& \text { Abbreviations and Acronyms } \\
& \qquad \begin{array}{ll}
\mathrm{AF} & =\text { atrial fibrillation } \\
\mathrm{AFL} & =\text { atrial flutter } \\
\mathrm{AT} & =\text { atrial tachycardia } \\
\mathrm{CHF} & =\text { congestive heart failure } \\
\mathrm{GP} & =\text { ganglionic plexi } \\
\mathrm{NYHA} & =\text { New York Heart Association } \\
\mathrm{RF} & =\text { radiofrequency } \\
\mathrm{SR} & =\text { sinus rhythm }
\end{array}
\end{aligned}
$$

can be eliminated with surgical bipolar radiofrequency (RF) isolation. ${ }^{9}$ On the other hand, Alex and Guvendik11 failed to demonstrate any role of cardiac denervation on AF recurrences after surgery. Different techniques aimed at reducing postoperative autonomic imbalance (eg, thoracic epidural anesthesia) also failed to reduce postoperative AF. ${ }^{11}$ Therefore, despite the postulated role for surgical GP isolation, there is no general agreement on the efficacy of strategies targeting the autonomic nervous system on postoperative $\mathrm{AF}^{7-10} \mathrm{Al}-$ though surgical AF ablation with modified "mini-Maze" procedures is now considered curative for the majority of patients referred to surgery, ${ }^{2}$ it still carries the risk for AF recurrence, estimated to range from $10 \%$ to $40 \%$ in the different series, during the short- and mid-term follow-ups. ${ }^{11,12} \mathrm{We}$ hypothesized that GP isolation with bipolar RF ablation may increase freedom from AF in patients undergoing persistent/long-standing persistent $\mathrm{AF}$ ablation during concomitant mitral valve surgery. The present study analyzes the hospital and short-term follow-up outcomes of patients undergoing 2 different surgical strategies (mini-Maze vs mini-Maze + GP isolation) used in the last 2.5 years to treat $\mathrm{AF}$ in patients undergoing mitral valve surgery.

\section{Materials and Methods}

From January of 2005 to October of 2007, 75 patients with persistent or longstanding persistent $\mathrm{AF}$ who required mitral valve surgery were enrolled in this prospective study after the approval of the institution's (Cardiac Surgery Unit Magna Graecia University) ethical committee/institutional review board and informed consent had been obtained. The study design was set to enroll patients undergoing the mini-Maze during mitral valve surgery in the first period (January of 2005 to September of 2006) and to compare hospital outcome and short-term follow-up with that of patients undergoing the combined mini-Maze + GP isolation and mitral valve surgery during the second period of the study (September of 2006 to October

\begin{tabular}{|c|c|c|c|}
\hline & $\begin{array}{c}\text { Group A } \\
\text { (44 patients) }\end{array}$ & $\begin{array}{c}\text { Group B } \\
\text { (31 patients) }\end{array}$ & $\boldsymbol{P}$ \\
\hline Age (y) & $64.6 \pm 10.3$ & $64.2 \pm 9.8$ & .849 \\
\hline Age $>65 y$ & $21(47.7 \%)$ & $21(67.7 \%)$ & .068 \\
\hline Sex (male) & $33(75.0 \%)$ & $23(74.2 \%)$ & .572 \\
\hline Diabetes mellitus & $14(31.8 \%)$ & $11(35.5 \%)$ & .465 \\
\hline Hypertension & $33(75.0 \%)$ & $22(71.0 \%)$ & .448 \\
\hline Dyslipidemia & $24(54.5 \%)$ & $13(41.9 \%)$ & .200 \\
\hline COPD & $19(43.2 \%)$ & $10(32.3 \%)$ & .238 \\
\hline$>90 \mathrm{~kg}$ (weight) & $12(27.3 \%)$ & $6(19.4 \%)$ & .306 \\
\hline Mitral disease & $44(100 \%)$ & $31(100 \%)$ & - \\
\hline Aortic disease & $22(50.0 \%)$ & $11(35.5 \%)$ & .156 \\
\hline Tricuspid disease & $16(36.4 \%)$ & $14(45.2 \%)$ & .299 \\
\hline Coronary heart disease & $5(11.4 \%)$ & $4(12.9 \%)$ & .556 \\
\hline Longstanding persistent AF & $38(86.4 \%)$ & $27(87.1 \%)$ & .605 \\
\hline Persistent AF & $6(13.6 \%)$ & $4(12.9 \%)$ & .605 \\
\hline $\mathrm{AF}$ duration (mo) & $29.1 \pm 10.7$ & $27.5 \pm 11.4$ & .548 \\
\hline Preoperative NYHA & $3.68 \pm 0.47$ & $3.58 \pm 0.62$ & .425 \\
\hline Preoperative heart failure state & $14(31.8 \%)$ & $11(35.5 \%)$ & .465 \\
\hline $\begin{array}{l}\text { Previous pharmacologic } \\
\text { cardioversion }\end{array}$ & $44(100 \%)$ & $31(100 \%)$ & - \\
\hline Previous electric cardioversion & $17(38.6 \%)$ & $8(25.8 \%)$ & .181 \\
\hline Preoperative LVEF & $45.6 \pm 10.4$ & $44.8 \pm 9.6$ & .738 \\
\hline Preoperative LVEF $<45 \%$ & $26(59.1 \%)$ & $17(54.8 \%)$ & .448 \\
\hline Preoperative transverse LAD & $53.7 \pm 6.7$ & $53.5 \pm 7.6$ & .906 \\
\hline Preoperative longitudinal LAD & $57.3 \pm 9.9$ & $59.2 \pm 12.6$ & .553 \\
\hline $\begin{array}{l}\text { Mitral valve replacement } \\
\text { (mechanical) }\end{array}$ & $7(15.9 \%)$ & $8(25.8 \%)$ & .222 \\
\hline $\begin{array}{l}\text { Mitral valve replacement } \\
\text { (biologic) }\end{array}$ & $4(9.1 \%)$ & - & .112 \\
\hline Mitral valve plasty & $33(75.0 \%)$ & $23(74.2 \%)$ & .572 \\
\hline $\begin{array}{l}\text { Aortic valve replacement } \\
\text { (mechanical) }\end{array}$ & $7(15.9 \%)$ & $4(12.9 \%)$ & .493 \\
\hline $\begin{array}{l}\text { Aortic valve replacement } \\
\text { (biologic) }\end{array}$ & $5(11.4 \%)$ & $2(6.5 \%)$ & .384 \\
\hline Aortic valve plasty & $3(6.8 \%)$ & $5(16.1 \%)$ & .182 \\
\hline Tricuspid valve plasty & $16(36.4 \%)$ & $14(45.2 \%)$ & .299 \\
\hline $\mathrm{CABG}$ & $5(11.4 \%)$ & $4(12.9 \%)$ & .556 \\
\hline Crossclamp time & $60.2 \pm 15.1$ & $59.1 \pm 11.3$ & .730 \\
\hline Cardiopulmonary bypass time & $83.7 \pm 12.5$ & $81.2 \pm 14.1$ & .407 \\
\hline
\end{tabular}
of 2007). AF was defined as persistent or longstanding persistent according to the Heart Rhythm Society, European Heart Rhythm Association, and European Cardiac Arrhythmia Society. ${ }^{14}$ The demographics are reported in Table 1.

\section{Surgery}

Cardiopulmonary bypass was standardized. ${ }^{13}$ Cardiac arrest was achieved with intermittent antegrade plus retrograde blood cardio-
TABLE 1. Demographic and intraoperative data

$A F$, Atrial fibrillation; $C O P D$, chronic obstructive pulmonary disease; $L A D$, left atrial diameter; $L V E F$, left ventricular ejection fraction; $N Y H A$, New York Heart Association; $C A B G$, coronary artery bypass grafting.

plegia. Mitral valve surgery was performed through a longitudinal atriotomy. Mitral valve replacement was always achieved by implanting prostheses in the intra-annular position. When tricuspid disease was encountered, longitudinal intercaval right atriotomy was used as the surgical access. Intraoperative data and concomitant procedures are also shown in Table 1 .

With regard to arrhythmia surgery, 2 different surgical strategies were adopted: A total of 44 patients underwent the isolated "left + right mini-Maze" procedure (group A) as previously reported, ${ }^{12}$ preferentially during the first phase of the study, and the remaining 31 patients (group B) underwent the "left + right mini-Maze" 
procedure plus GP isolation, preferentially during the second phase of the study. Briefly, left-sided lesions were in the pulmonary veins and left atrial appendage bipolar RF isolation, with the bipolar clamp (Medtronic, Minneapolis, Minn) and Cardioblate surgical ablation generator (Medtronic) as the power source. The left isthmus lesion was always performed with a unipolar RF pen (Cardioblate surgical pen; Medtronic). The left lesion set was completed by bipolar RF lesion connecting the left upper pulmonary vein to the left atrial appendage. Right-sided ablation lines were created as previously reported, always using a bipolar clamp. ${ }^{12}$ Briefly, a purse-string suture stitched in front of the coronary sinus was used to insert the bipolar clamp, which was first directed between the inferior vena cava and the coronary sinus, and then rotated toward the atrioventricular groove, between the coronary sinus and the tricuspid valve, to ablate the cavo-tricuspid isthmus. The clamp was subsequently rotated upward to create a transmural ablation line, connecting the purse string to the right appendage. Finally, an additional purse-string suture was stitched on the tip of the right atrial appendage (for venous drainage), through which the bipolar clamp was introduced to first allow an ablative line connecting the superior vena cava to the inferior vena cava and then to ablate the terminal crest. Off-pump ablation of the right appendage completed the procedure. ${ }^{12}$ Because of the risk of incomplete right septal isthmus ablation, patients with a large right atrium $(\geq 7.0 \mathrm{~cm})$ undergoing tricuspid valvuloplasty also underwent further monopolar RF septal isthmus ablation. Left and right appendages were always excluded by external ligation or internal stitching. ${ }^{12}$

\section{Ganglionic Plexi Isolation}

In patients undergoing GP isolation, intraoperative epicardial electrophysiologic mapping was performed at the time of operation, starting from the right-sided pulmonary veins, moving from the area of the superior right pulmonary vein to that of the inferior right pulmonary vein, and then moving in the same manner to the leftsided pulmonary veins. Intraoperative epicardial electrophysiologic mapping was performed using a standard 6F catheter (FIAB SpA, Florence, Italy). The catheter was connected to an external pulse generator (St Jude Medical Inc, St Paul, Minn) to deliver highfrequency stimulation (at a rate of 800/min, amplitude of 12 to 16 $\mathrm{mA}$, with a pulse duration of $9.9 \mathrm{~ms}$ ) to the above-mentioned areas. The electrocardiogram was monitored, and stimulation that resulted in a doubling of the R-R interval indicated an active GP. ${ }^{9}$ A bipolar $\mathrm{RF}$ lesion encompassing the right-sided interatrial fat pad of Waterston's groove, as suggested by Mehall and coworkers, ${ }^{9}$ was then made. This lesion was created with 1 jaw of the bipolar clamp posterior to the right-sided pulmonary veins and the other jaw on the right atrium, medial to the interatrial fat pad, to encompass part of the left atrium, right atrium, and interatrial septum. Another bipolar RF clamping was made jawing the ligament of Marshall. ${ }^{9}$ GP areas that had preablation activity were then restimulated to confirm the isolation. Both lesions involving GP locations were made off pump and at least twice to achieve isolation. Extended fat pat resection along Waterston's groove, the ligament of Marshall, and the left-sided pulmonary veins was then executed to demonstrate GP with pathologic analysis on the specimens. Each right- and left-sided fat pad specimen was divided into 3 portions (superior, middle, and inferior), and active ganglia were localized in each specimen. To differentiate ganglia from nerves, specimens were stained with hematoxylin/eosin and antibodies to S100 protein and neurofilaments.
However, except for the surgical AF ablation technique, preoperative, intraoperative, and postoperative management techniques were standard during the entire study period.

\section{Postoperative Care}

Inotropic support was defined as low dose when enoximone was administered at a dosage of $5 \mu \mathrm{g} / \mathrm{kg} / \mathrm{min}$ or less, medium dose when enoximone was administered at a dosage of 6 to $10 \mu \mathrm{g} / \mathrm{kg} / \mathrm{min}$ or dobutamine was added from 5 to $10 \mu \mathrm{g} / \mathrm{kg} / \mathrm{min}$, and high dose when enoximone or dobutamine was administered at a dosage of $10 \mu \mathrm{g} /$ $\mathrm{kg} / \mathrm{min}$ or epinephrine at any dose was added.

An intravenous amiodarone bolus $(150 \mathrm{mg})$, followed by continuous intravenous infusion at $1 \mathrm{mg} / \mathrm{kg} / \mathrm{h}$ for 12 hours and then 0.5 $\mathrm{mg} / \mathrm{kg} / \mathrm{h}$ until patients tolerated oral intake, was routinely administered intraoperatively. Patients then underwent oral amiodarone (200 mg twice per day for 1 week, then $200 \mathrm{mg}$ daily) until the sixth postoperative month. Patients with postoperative persistence of AF, as well as those with new-onset AF, did not undergo electrical cardioversion but were treated with intravenously and orally administered amiodarone. The international normalized ratio was maintained between 2.5 and 3.5 for the first 6 months in all patients and lifelong in patients receiving mechanical valves or with AF persistence.

\section{Assessment of Cardiac Rhythm and Follow-up}

Twelve-lead electrocardiographic recordings were performed preoperatively, on admission to the intensive therapy unit, and daily thereafter until hospital discharge or whenever necessary. All patients underwent continuous electrocardiographic monitoring for the first 72 hours postoperatively. The incidence of arrhythmias, both atrial and ventricular, was recorded during hospitalization and follow-up. The mean follow-up time was $16.7 \pm 0.95$ (standard error) months (confidence limit 14.9-18.6 months), and no patient was lost during follow-up (100\% complete). An initial post-ablation blanking period of 3 months was considered according to the Heart Rhythm Society, European Heart Rhythm Association, and European Cardiac Arrhythmia Society: The reported recurrences during the post-ablation blanking period were defined as "early events.",11 Follow-up data were collected by routine examination in our outpatient clinic ( 7 days, 14 days, 1 month, 3 months, 6 months, and 1 year after surgery, and then once per year or whenever requested by the patient). Phone contacts with patients and cardiologists were made whenever necessary. One month after surgery, patients underwent 24-hour Holter electrocardiography, which was repeated at least once during the first 6 months of follow-up. According to the Heart Rhythm Society, European Heart Rhythm Association, and European Cardiac Arrhythmia Society, 6- and 12-month followups were recorded. Follow-up was closed on November 30, 2007.

\section{End Points}

The primary efficacy end point was freedom from AF, atrial flutter (AFL), and atrial tachycardia (AT) of at least 30 seconds duration in the absence of antiarrhythmic drug therapy 3 months after stopping amiodarone. ${ }^{11}$ The prevalence of AF at 6 and 12 months postoperatively were also recorded. Secondary end points were freedom from AF, AFL, and AT in the presence of antiarrhythmic therapy, prevalence of "early events" during the post-ablation blanking period of 3 months, in-hospital electrophysiologic results, and freedom from episodes of congestive heart failure (CHF) and hospital readmission. CHF was defined as any episode of cardiac 
decompensation during follow-up requiring adjustment of the therapy or hospitalization; hospital readmission was defined as any hospital admission for any cardiac cause and requiring more than a 6-hour stay. As a surrogate outcome of cardiac compensation, the need and dose of furosemide were registered preoperatively, at hospital discharge, and at the end of follow-up.

\section{Echocardiography}

The echocardiographic examinations were performed preoperatively, postoperatively, and at 6 months follow-up using a VIVID 7 Pro ultrasound machine (GE Technologies, Milwaukee, Wis). Atrial and ventricular remodeling (longitudinal and transverse atrial diameters, diastolic ventricular diameter), systolic pulmonary arterial pressure, indexed left ventricular mass, and E/A ratio recovery were investigated.

\section{Statistical Analysis}

Continuous variables are expressed as mean \pm standard deviation, and categoric data are expressed as proportions. Comparisons of continuous variables were made with the Student unpaired $t$ test, and categoric variables were compared with the chi-square test or Fisher exact test. Comparison between and within groups was made using 2-way analysis of variance for repeated measures.

Freedom from AF, AFL, AT, CHF, and hospital readmission was determined by means of the Kaplan-Meier life-table analysis. Log-rank test was performed to ascertain differences during follow-up between patients undergoing the isolated left + right miniMaze procedure and those undergoing combined GP isolation. All statistical analyses were performed with the Statistical Package for the Social Sciences 13.0 (SPSS Inc, Chicago, Ill).

\section{Results}

\section{Hospital Mortality, Morbidity, and Drug-related Complications}

There were no hospital deaths during hospitalization or ablation device-related complications. Hospital morbidity is reported in Table 2. No differences were registered in perioperative inotropic support (Table 2).

TABLE 2. Hospital morbidity and perioperative inotropic support

\begin{tabular}{lccc}
\hline & $\begin{array}{c}\text { Group A } \\
\text { (44 patients) }\end{array}$ & $\begin{array}{c}\text { Group B } \\
\text { (31 patients) }\end{array}$ & $\boldsymbol{P}$ \\
\hline Morbidity & $7 / 44(15.9 \%)$ & $6 / 31(19.4 \%)$ & .464 \\
LOS & $2 / 44(4.5 \%)$ & $2 / 31(6.5 \%)$ & .551 \\
ARF & $3 / 44(6.8 \%)$ & $2 / 31(6.5 \%)$ & .664 \\
Pneumonia & $2 / 44(4.5 \%)$ & $2 / 31(6.5 \%)$ & .551 \\
LD inotropic support & $24 / 44(54.5 \%)$ & $18 / 31(58.1 \%)$ & .474 \\
MD inotropic support & $16 / 44(36.4 \%)$ & $11 / 31(35.5 \%)$ & .567 \\
HD inotropic support & $4 / 44(9.1 \%)$ & $2 / 31(6.5 \%)$ & .516 \\
ITU stay (h) & $51.6 \pm 9.6$ & $50.2 \pm 6.5$ & .481 \\
Hospital stay (d) & $9.0 \pm 12.5$ & $7.3 \pm 0.8$ & .481 \\
\hline
\end{tabular}

$L O S$, Low output syndrome; $A R F$, acute renal failure; $L D$, low dose; $M D$, medium dose; $H D$, high dose; ITU, intensive therapy unit.
When chronic amiodarone therapy was considered, only 1 patient in group A $(2.3 \% ; P=.587)$ experienced optic neuropathy at 4 months after surgery, with partial improvement after drug discontinuation. However, all other patients continued amiodarone until the sixth month. Finally, no warfarin-related complications were registered during the study.

\section{Pathologic Analysis}

The details of the site and number of GPs detected at pathology are shown in Table 3. As evidenced, GPs are generally clustered in a higher number on the upper right and left fat pad specimens. No GP can be demonstrated in 1 superior, 6 middle, and 12 inferior right fat pad specimens, and in 11 superior, 4 middle, and 7 inferior left fat pad specimens (Table 3). Obviously, none of these specimens were active at intraoperative electrophysiologic analysis. A mean of $3.09 \pm$ $1.13 \mathrm{GP}$ (range $0-5$ ) in the superior, $1.70 \pm 1.04 \mathrm{GP}$ (range

TABLE 3. Number of ganglionic plexi found at pathologic analysis in each patient

\begin{tabular}{lcccccc}
\hline & $\begin{array}{c}\text { Superior } \\
\text { right FPS }\end{array}$ & $\begin{array}{c}\text { Middle } \\
\text { right FPS }\end{array}$ & $\begin{array}{c}\text { Inferior } \\
\text { right FPS }\end{array}$ & $\begin{array}{c}\text { Superior } \\
\text { left FPS }\end{array}$ & $\begin{array}{c}\text { Middle } \\
\text { left FPS }\end{array}$ & $\begin{array}{c}\text { Inferior } \\
\text { left FPS }\end{array}$ \\
\hline Patient 1 & 4.00 & 3.00 & 3.00 & 3.00 & 3.00 & 3.00 \\
Patient 2 & 3.00 & 2.00 & 2.00 & 3.00 & 3.00 & 2.00 \\
Patient 3 & 3.00 & 2.00 & 3.00 & 2.00 & 3.00 & 2.00 \\
Patient 4 & 4.00 & 2.00 & 2.00 & 2.00 & 2.00 & 2.00 \\
Patient 5 & 4.00 & 3.00 & 2.00 & 4.00 & 2.00 & 1.00 \\
Patient 6 & 4.00 & 2.00 & 3.00 & 3.00 & 2.00 & 1.00 \\
Patient 7 & 3.00 & 2.00 & 2.00 & 2.00 & 2.00 & 1.00 \\
Patient 8 & 3.00 & 2.00 & 1.00 & 2.00 & 2.00 & 1.00 \\
Patient 9 & 3.00 & 3.00 & 1.00 & 3.00 & 1.00 & 1.00 \\
Patient 10 & 3.00 & 2.00 & 0.00 & 3.00 & 1.00 & 1.00 \\
Patient 11 & 3.00 & 2.00 & 2.00 & 1.00 & 1.00 & 1.00 \\
Patient 12 & 3.00 & 2.00 & 1.00 & 1.00 & 1.00 & 1.00 \\
Patient 13 & 4.00 & 1.00 & 2.00 & 0.00 & 1.00 & 0.00 \\
Patient 14 & 3.00 & 3.00 & 2.00 & 0.00 & 1.00 & 2.00 \\
Patient 15 & 3.00 & 3.00 & 2.00 & 0.00 & 1.00 & 1.00 \\
Patient 16 & 3.00 & 1.00 & 0.00 & 0.00 & 1.00 & 1.00 \\
Patient 17 & 3.00 & 2.00 & 0.00 & 0.00 & 1.00 & 1.00 \\
Patient 18 & 4.00 & 2.00 & 0.00 & 0.00 & 1.00 & 1.00 \\
Patient 19 & 4.00 & 0.00 & 1.00 & 0.00 & 1.00 & 0.00 \\
Patient 20 & 5.00 & 2.00 & 1.00 & 1.00 & 1.00 & 1.00 \\
Patient 21 & 4.00 & 3.00 & 2.00 & 1.00 & 1.00 & 1.00 \\
Patient 22 & 4.00 & 3.00 & 1.00 & 1.00 & 1.00 & 1.00 \\
Patient 23 & 5.00 & 2.00 & 1.00 & 1.00 & 1.00 & 1.00 \\
Patient 24 & 3.00 & 2.00 & 0.00 & 1.00 & 1.00 & 1.00 \\
Patient 25 & 3.00 & 1.00 & 0.00 & 1.00 & 1.00 & 1.00 \\
Patient 26 & 3.00 & 1.00 & 0.00 & 0.00 & 1.00 & 1.00 \\
Patient 27 & 1.00 & 0.00 & 0.00 & 2.00 & 1.00 & 0.00 \\
Patient 28 & 1.00 & 0.00 & 0.00 & 0.00 & 0.00 & 0.00 \\
Patient 29 & 0.00 & 0.00 & 0.00 & 1.00 & 0.00 & 0.00 \\
Patient 30 & 1.00 & 0.00 & 0.00 & 0.00 & 0.00 & 0.00 \\
Patient 31 & 2.00 & 0.00 & 0.00 & 0.00 & 0.00 & 0.00 \\
\hline FPS,Fat pad & & & & & & \\
\hline
\end{tabular}

FPS, Fat pad specimen. 
$0-3$ ) in the middle, and $1.09 \pm 1.04 \mathrm{GP}$ (range $0-3$ ) in the inferior right fat pad specimens were demonstrated. Similarly, a mean of $1.22 \pm 1.20$ GP (range $0-4), 1.22 \pm 0.80$ GP (range 0-3), and $0.96 \pm 0.70$ GP (range $0-3$ ) were detected in the superior, middle, and inferior left fat pad specimens, respectively.

On the other hand, the prevalence of active sites along the fat pad specimens, as demonstrated by intraoperative electrophysiologic mapping, is shown in Figure 1. Inactive zones did not undergo further electrophysiologic stimulation with different frequencies, pulse durations, or amplitudes. Active sites were found bilaterally in 16 patients, unilaterally right sided in 8 patients, and left sided in 7 patients. When active fat pad specimens were compared with inactive fat pad specimens, a significantly higher number of GP were detected by the pathologist in active specimens (superior right: $3.54 \pm$ 0.65 vs $1.57 \pm 1.13$ in inactive specimens, $P=.003$; middle right: $2.16 \pm 0.61$ vs $1.07 \pm 1.18, P=.008$; inferior right: $2.42 \pm 0.53$ vs $0.70 \pm 0.80, P=.0001$; superior left: 2.70 \pm 0.67 vs $0.52 \pm 0.60, P=.0001$; middle left: $2.37 \pm$ 0.51 vs $0.82 \pm 0.38, P=.0001$; inferior left: $2.0 \pm 0.70$ vs $0.76 \pm 0.51, P=.015)$. All patients in whom AF recurrences developed during follow-up demonstrated at least 1 active fat pad zone. However, no differences were found in the number and site of GP in patients demonstrating AF recurrences during follow-up compared with patients maintaining SR (superior right: AF $3.00 \pm 1.41$ vs SR $3.11 \pm 1.12$, $P=.859$; middle right: $1.25 \pm 1.25$ vs $1.78 \pm 1.01, P=$
.352 ; inferior right: $1.25 \pm 1.50$ vs $1.07 \pm 0.99, P=.759$; superior left: $1.00 \pm 1.41$ vs $1.25 \pm 1.19, P=.695$; middle left: $1.25 \pm 1.26$ vs $1.22 \pm 0.75, P=.950$; inferior left: 1.00 \pm 1.41 vs $0.96 \pm 0.58, P=.962$ ).

\section{Cardiac Rhythm}

On aortic declamping, 51 patients $(68.0 \%)$ recovered SR, 14 patients $(18.7 \%)$ were in junctional rhythm, and 10 patients (13.3\%) displayed persistence of AF. Only 1 case of AF persistence was registered at aortic declamping in group $\mathrm{B}$, and 9 cases were registered in group A (20.5\% vs group B $3.2 \%$; $P=.030)$. No differences were recorded between the 2 groups in SR (30/44 [68.2\%] vs group B: 21/31 [67.7\%]; $P$ $=.581)$ and junctional rhythm requiring temporary pacemaker stimulation (5/44 [11.4\%] vs group B: 9/31 [29.0\%]; $P=.052)$. Of these, only 1 patient $(1.3 \%$ of the global population) in group A $(2.3 \%$ vs group B $0 \% ; P=.587)$ required definitive pacemaker implantation.

All patients were treated with amiodarone postoperatively; thus, 64 patients $(85.3 \%)$ recovered and were discharged in SR, and 10 patients (13.3\%) were discharged in AF. With regard to AFL, postoperative transient AFL developed during hospitalization in 11 patients $(14.7 \%)$, without differences between the 2 groups (group A: 7/44 [15.9\%] vs group B: $4 / 31$ [12.9\%]; $P=.493$ ). On discharge, a significantly higher prevalence of AF was noted in group A (9/44 [20.5\%] vs group B: $1 / 31$ [3.2\%]; $P=.030$ ), together with a lower prevalence of SR (group A: 34/44 [77.3\%] vs group
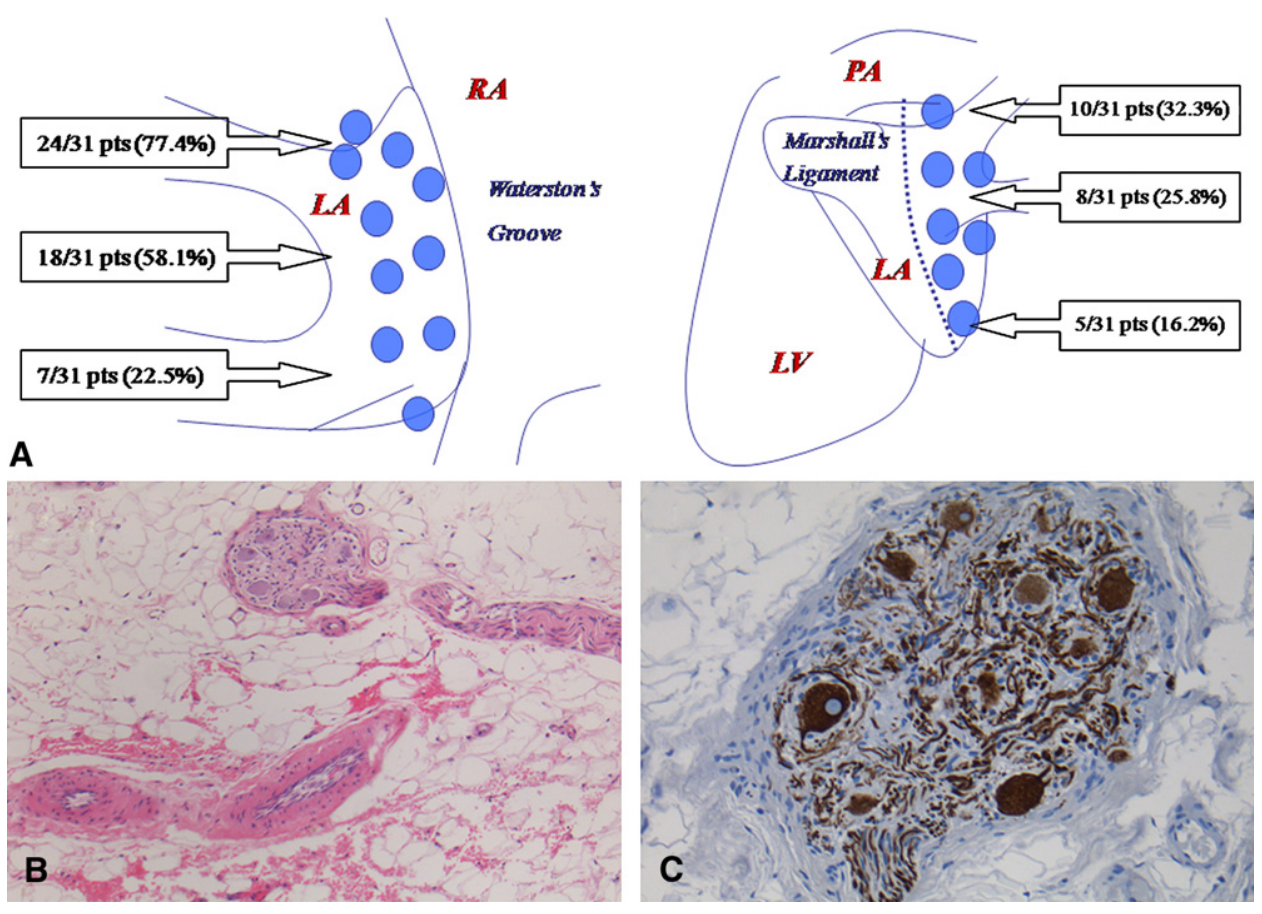

Figure 1. A, Electrophysiologic mapping of active GP. GP demonstration on fat pad specimen stained with hematoxylin/eosin (B) and antibodies to neurofilaments (C). $L A$, Left atrium; $R A$, right atrium; $P A$, pulmonary artery; $L V$, left ventricle. 
B: 30/31 [96.8\%]; $P=.01)$. No differences were recorded in "early events" during the post-ablation blanking period of 3 months between the 2 groups (group A: 5/44 [11.4\%] vs group $\mathrm{B}: 3 / 31$ [9.7\%]; $P=.565)$. After antiarrhythmic therapy discontinuation, no differences in the prevalence of $\mathrm{AF}$, AFL, and AT were registered at 6 months (group A: 4/36$11.1 \%$ vs group $\mathrm{B}: 1 / 29-3.4 \% ; P=.252$ ), but a significantly lower AF, AFL, and AT prevalence was recorded in group B at 12 months $(1 / 11$ [9.1\%] vs group A:14/33 [42.4\%]; $P=$ .044 ). At a mean of $11.7 \pm 5.9$ months follow-up (range 1-22 months), 57 patients $(76.0 \%)$ were in SR. Actuarial freedom from AF, AFL, and AT recurrence with antiarrhythmic therapy after $16.7 \pm 0.95$ (standard error) months was $63.2 \% \pm 7.3 \%$ (Figure 2, $A$ ) and proved higher in group B (Figure 2, B). However, after the sixth month, $12.8 \pm 0.80$ (standard error) months, freedom from AF, AFL, and AT without antiarrhythmic therapy was $72.5 \% \pm 7.7 \%$ (Figure 2, $C$ ) higher in group B (Figure 2, D).
When AF-surgery related complications were considered, no cases of cardiac tamponade, pulmonary vein stenosis, esophageal injury, phrenic/vagal nerve injury, coronary occlusions, or thromboembolism were registered.

\section{Hemodynamic Status, Congestive Heart Failure, and Hospital Readmission}

New York Heart Association (NYHA) class improved significantly after surgery within the 2 groups (group A preoperative: $3.68 \pm 0.47$ vs discharge: $1.45 \pm 0.58, P=.0001$; group B preoperative: $3.58 \pm 0.62$ vs discharge: $1.35 \pm$ $0.48, P=.0001)$ and further ameliorated during follow-up (group A follow-up NYHA: $1.16 \pm 0.48, P=.0001$; group B follow-up NYHA: $1.19 \pm 0.40, P=.0007$; statistical significance between groups: $P=.517)$. On the other hand, patients with AF displayed less improvement in NYHA class than those with SR at discharge and during follow-up (preoperative NYHA of patients with AF: $3.70 \pm 0.46$ vs NYHA on

Freedom from AFIAFLIAT with antiarrhythmic therapy - global population

Freedom from AFIAFLIAT with antiarrhythmic therapy. Group A vs Group B
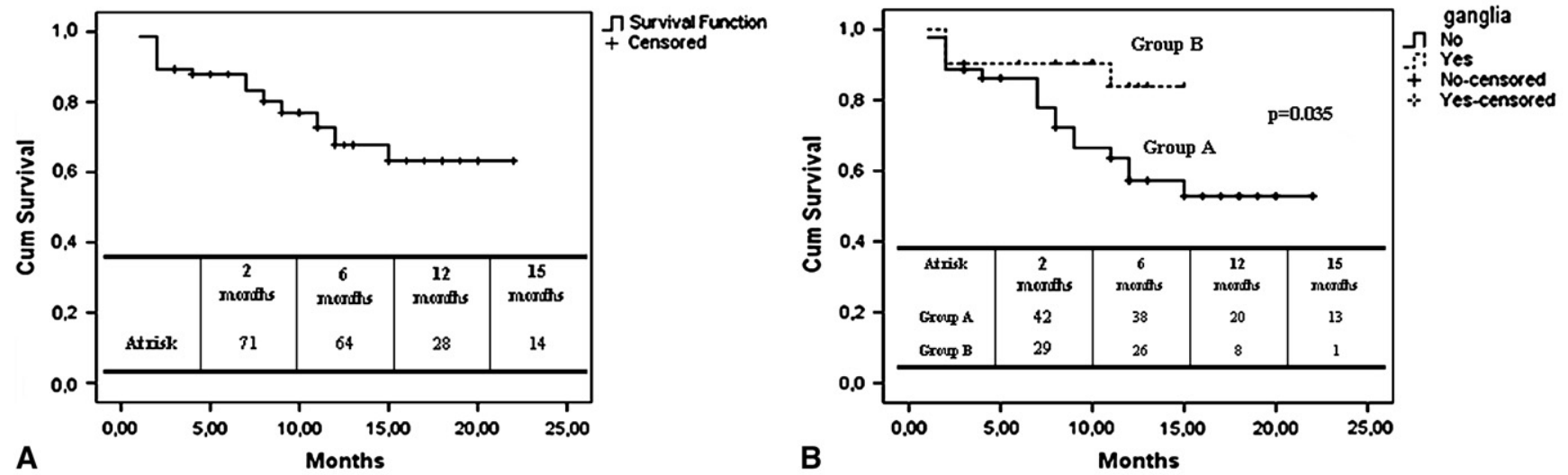

Freedom from AFIAFLIAT without antiarrhythmic therapy - global population Freedom from AFIAFLIAT without antiarrhythmic therapy-Group A vs Group B
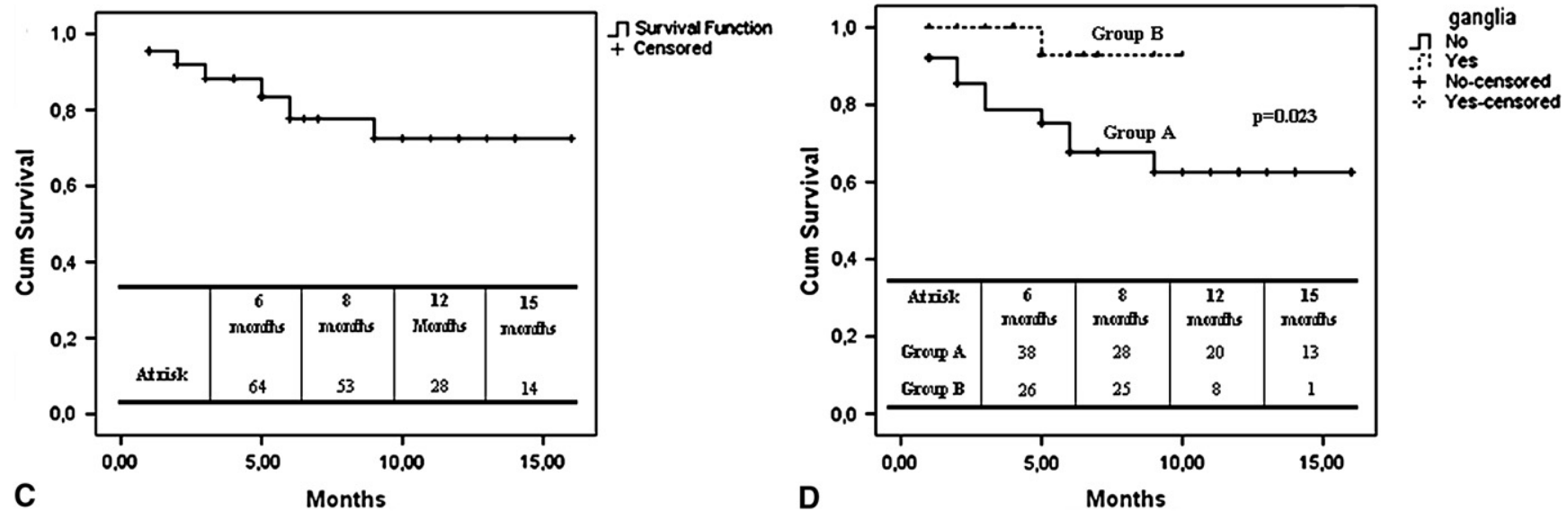

Figure 2. Cumulative freedom from $A F, A F L$, and $A T$ with antiarrhythmic therapy (A), freedom from AF, AFL, and AT with antiarrhythmic therapy between the 2 groups $(B)$, cumulative freedom from AF, AFL, and AT without antiarrhythmic therapy (C), and freedom from AF, AFL, and AT without antiarrhythmic therapy between the 2 groups (D). AF, Atrial fibrillation; $A F L$, atrial flutter; $A T$, atrial tachycardia. 
discharge: $1.76 \pm 0.66$ vs follow-up NYHA: $1.52 \pm 0.62$, statistical probability within subjects $P=.040$; preoperative NYHA of patients with SR: $3.62 \pm 0.55$ vs NYHA on discharge: $1.31 \pm 0.46$ vs follow-up NYHA: $1.06 \pm 031$, statistical probability within subjects $P=.0001$; statistical probability between groups $P=.001)$. When furosemide therapy was considered, of 42 patients in group A (95.5\%) and 31 patients in group B $(100 \% ; P=.341)$, who were taking diuretics on hospital admission, 41 patients in group A $(93.2 \%)$ and 29 patients in group B $(93.5 \% ; P=.664)$ were discharged with furosemide. Of these, 22 patients in group A $(50.0 \%)$ and 17 patients in group B $(54.8 \% ; P=$ .430) continued to take diuretics at the end of follow-up. However, a significantly higher diuretic dose was necessary in group A (preoperative: $51.7 \pm 24.8 \mathrm{mg} / \mathrm{d}$ vs discharge: $55.1 \pm 20.1 \mathrm{mg} / \mathrm{d}$ vs follow-up: $19.6 \pm 16.6 ; P=.070$ within group) compared with group B (preoperative: $70.2 \pm 26.1$ $\mathrm{mg} / \mathrm{d}$ vs discharge: $32.2 \pm 16.6 \mathrm{mg} / \mathrm{d}$ vs follow-up: $13.7 \pm$ 13.8; $P=.0001$ within group; statistical significance between groups: $P=.0001)$. Moreover, patients maintaining SR at discharge and during follow-up needed less diuretics than patients experiencing AF recurrence, either in group A (SR at discharge: $36.3 \pm 22.2 \mathrm{mg} / \mathrm{d}$ vs AF at discharge: $100.0 \pm 51.1 ; P=.0001 ;$ SR at follow-up: $9.6 \pm 11.9 \mathrm{mg} /$ $\mathrm{d}$ vs AF at follow-up: $43.2 \pm 34.8 ; P=.0001$; statistical significance between groups: $P=.0001$ ) or group B (SR at discharge: $28.7 \pm 13.3 \mathrm{mg} / \mathrm{d}$ vs AF at discharge: $56.2 \pm 12.5$; $P=.001 ;$ SR at follow-up: $11.1 \pm 12.2 \mathrm{mg} / \mathrm{d}$ vs AF at follow-up: $31.2 \pm 12.5 ; P=.005$; statistical significance between groups: $P=.003$ ).

Seven patients in group A had episodes of CHF during follow-up, giving a global actuarial freedom from $\mathrm{CHF}$ of $84.0 \% \pm 6.4 \%$ (Figure 3, A). However, no differences were recorded between the 2 groups in follow-up freedom from CHF (Figure 3, B). On the other hand, 8 patients were readmitted to hospital during follow-up, meaning that cumulative actuarial freedom from hospital readmission was $87.3 \% \pm$ $4.3 \%$ (Figure 3, C). Again, no differences were detected

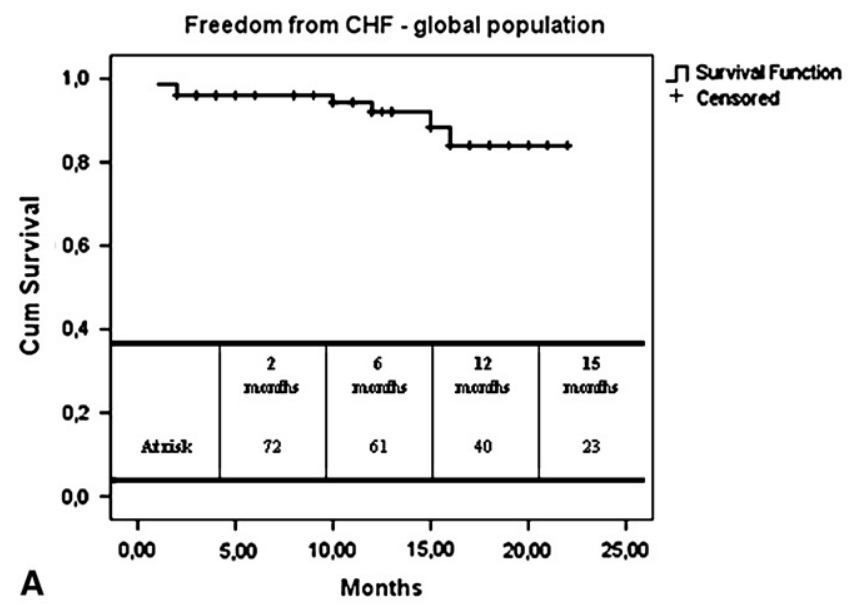

Freedom from Hospital Readmission - global population
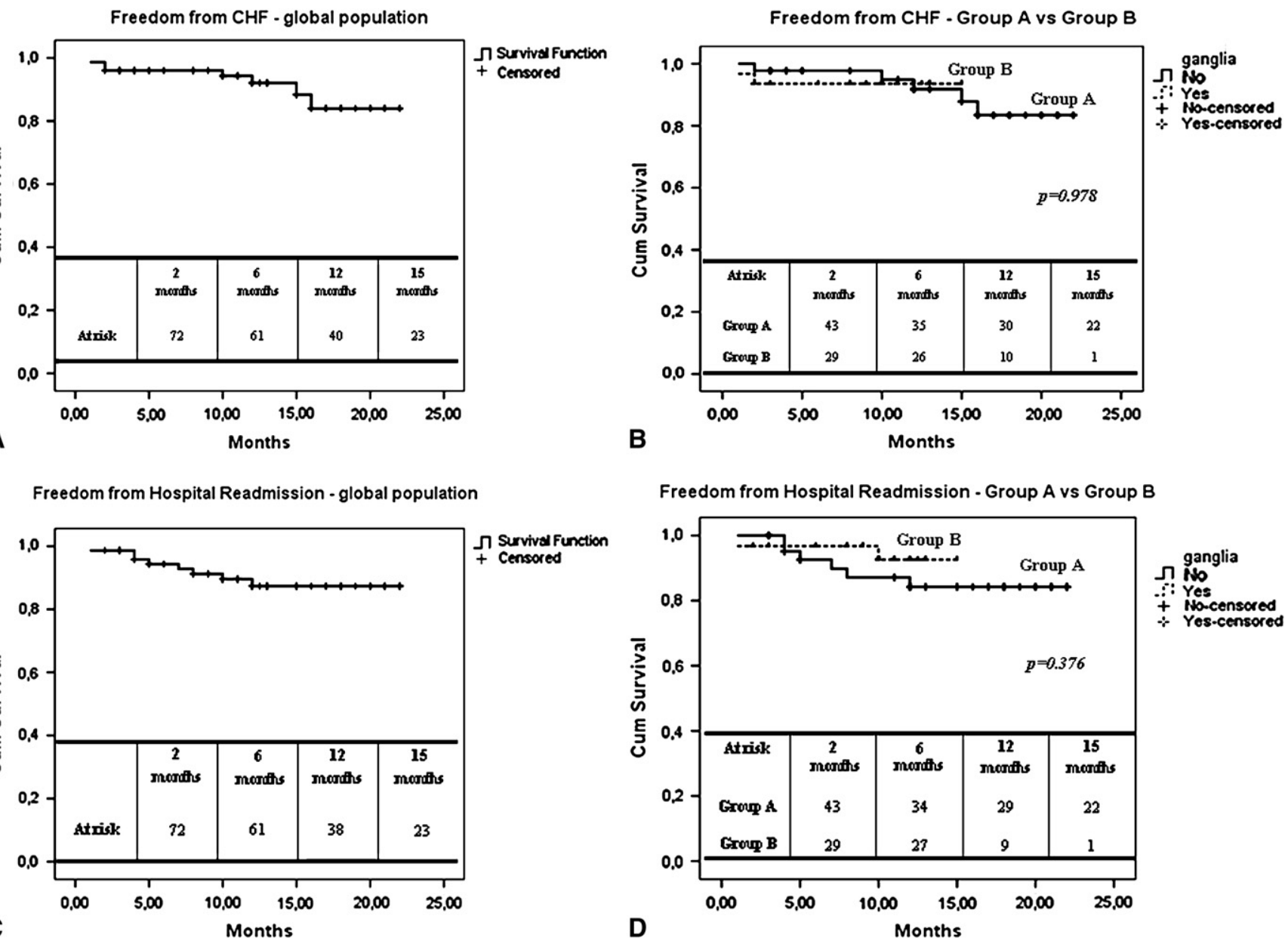

Figure 3. Cumulative freedom from CHF (A), freedom from CHF between the 2 groups (B), cumulative freedom from hospital readmission (C), and freedom from hospital readmission between the 2 groups (D). CHF, Congestive heart failure. 
between group A and B in follow-up freedom from hospital readmission (Figure $3, D$ ).

\section{Echocardiographic Results}

Echocardiography documented a normalized E/A in 35 patients $(46.7 \%$ of global population, $61.4 \%$ of patients with $\mathrm{SR}$ ) at the end of follow-up. Patients undergoing GP isolation demonstrated a higher prevalence of normalized E/A (group B: $19 / 31$ [61.3\%] vs group A: 16/44 [36.4\%], $P=.029 ; 19 /$ 27 patients with SR [70.4\%] vs $16 / 30$ patients with SR [53.3\%], $P=.148)$.

When groups A and B were compared, no differences in follow-up echocardiographic parameters were detected; both groups showed significant reverse remodeling of longitudinal and transverse atrial diameters, recovery of systolic pulmonary arterial pressure and indexed left ventricular mass, and follow-up reverse remodeling of diastolic left ventricular diameter (Table 4).

However, patients who recovered SR displayed significant reverse remodeling of left atrial longitudinal and transverse diameters on follow-up echocardiography (Table 4). Such reverse remodeling was not detected in patients in whom AF persisted (Table 4). Only patients in SR showed significant reverse remodeling of left ventricular diastolic diameter during follow-up, as well as a significant improve-

TABLE 4. Echocardiographic results stratified by cardiac rhythm and surgical treatment

\begin{tabular}{|c|c|c|c|c|c|}
\hline & & Discharge & Follow-up & $P^{\mathbf{a}}$ & $P^{\mathrm{b}}$ \\
\hline \multirow[t]{2}{*}{ Longitudinal LAD } & SR & $58.4 \pm 7.0$ & $51.7 \pm 7.4$ & .0001 & .0001 \\
\hline & $\mathrm{AF}$ & $61.5 \pm 9.4$ & $60.8 \pm 9.5$ & .120 & \\
\hline \multirow[t]{2}{*}{ Transverse LAD } & SR & $53.5 \pm 7.7$ & $47.9 \pm 8.1$ & .001 & .001 \\
\hline & $\mathrm{AF}$ & $54.4 \pm 7.5$ & $54.0 \pm 8.2$ & .181 & \\
\hline \multirow[t]{2}{*}{ PAPs } & SR & $33.2 \pm 2.5$ & $27.4 \pm 3.2$ & .008 & .005 \\
\hline & $\mathrm{AF}$ & $32.7 \pm 3.8$ & $31.4 \pm 3.2$ & .521 & \\
\hline \multirow[t]{2}{*}{ LVMi } & SR & $146.9 \pm 31.3$ & $130.1 \pm 29.3$ & .0001 & .08 \\
\hline & $\mathrm{AF}$ & $135.8 \pm 26.2$ & $125.4 \pm 25.6$ & .001 & \\
\hline \multirow[t]{2}{*}{ LVDd } & SR & $5.6 \pm 1.3$ & $5.2 \pm 1.2$ & .0001 & .001 \\
\hline & $\mathrm{AF}$ & $5.5 \pm 1.0$ & $5.4 \pm 1.2$ & .171 & \\
\hline \multirow[t]{2}{*}{ Longitudinal LAD } & Group A & $53.4 \pm 11.4$ & $51.0 \pm 9.2$ & .0001 & .465 \\
\hline & Group B & $54.9 \pm 10.8$ & $52.9 \pm 8.8$ & .0001 & \\
\hline \multirow[t]{2}{*}{ Transverse LAD } & Group A & $52.5 \pm 9.4$ & $51.3 \pm 7.7$ & .004 & .543 \\
\hline & Group B & $53.8 \pm 9.9$ & $51.0 \pm 8.0$ & .009 & \\
\hline \multirow[t]{2}{*}{ PAPs } & Group A & $32.6 \pm 8.4$ & $28.6 \pm 9.9$ & .015 & .426 \\
\hline & Group B & $33.4 \pm 9.2$ & $30.4 \pm 10.4$ & .034 & \\
\hline \multirow[t]{2}{*}{ LVMi } & Group A & $144.6 \pm 31.2$ & $123.9 \pm 28.1$ & .0001 & .454 \\
\hline & Group B & $142.5 \pm 29.1$ & $125.8 \pm 27.4$ & .0001 & \\
\hline \multirow[t]{2}{*}{ LVDd } & Group A & $5.4 \pm 1.0$ & $5.0 \pm 1.4$ & .0001 & .377 \\
\hline & Group B & $5.5 \pm 1.3$ & $5.2 \pm 1.0$ & .0001 & \\
\hline
\end{tabular}

$\angle A D$, Left atrial diameter; $P A P s$, systolic pulmonary arterial pressure; $L V M i$, indexed left ventricular mass; $L V D d$, diastolic left ventricular diameter. ${ }^{\text {a }}$ Statistical significance between discharge and follow-up echocardiography (within groups). ${ }^{b}$ Statistical significance between patients with SR and patients with $A F$ (first part) and between group $A$ and $B$ (second part) (between groups). ment in systolic pulmonary arterial pressure and indexed left ventricular mass (Table 4). In contrast, patients with AF only showed a significant improvement in follow-up indexed left ventricular mass (Table 4).

\section{Discussion}

Although it has long been ascertained that AF is frequently associated in patients with underlying heart disease, inasmuch as it is estimated to be present in $50 \%$ of patients with mitral valve disease, routine ablation of AF in these patients is a recent phenomenon. ${ }^{2}$ The Cox maze III operation is considered the gold standard for surgical treatment of AF, and other approaches to AF ablation should be measured against it. ${ }^{1-3}$ In this surgical procedure, multiple left and right atrial incisions are placed to interrupt the multiple reentrant circuits of AF. ${ }^{1}$ However, the Cox-Maze procedure is a technically demanding operation with a prolonged operative time, so that a number of modified "mini-Maze" procedures with alternative energy sources, other than the cut-and-sew technique, have been proposed to overcome such matters. ${ }^{2,11}$ However, if the Cox-Maze technique allows an SR restoration rate of $70 \%$ to $95 \%$ in the different experiences, ${ }^{2,14,15}$ the modified so-called mini-Maze procedures have a similar success rate ranging from $65 \%$ to $90 \%$, regardless of the ablative pattern, patient populations, and energy sources. ${ }^{2,16-18}$

All of these data imply that AF recurrences developed during the follow-up in approximately $25 \%$ of patients undergoing surgical AF ablation, limiting the primary surgical success rate. Therefore, despite the fact that Haissaguerre 4 demonstrated the importance of ectopic triggers of the pulmonary veins, different mechanisms can be involved in AF initiation and recurrence. In the last few years, different studies have demonstrated a significant role of parasympathetic autonomic tone in determining AF. ${ }^{5-9,19}$ Active GP are usually located in epicardial fat pads along the right-sided interatrial groove and along the ligament of Marshall on the left. ${ }^{9}$ Therefore, their location is often outside the areas traditionally isolated when pulmonary vein isolation alone is performed. ${ }^{9}$ In particular, it has been demonstrated that GP play a synergistic role with ectopic vein foci, thus decreasing the threshold of inducibility for AF. ${ }^{6}$ GP activation resulted in a progressive decrease in atrial refractoriness, which remains active even after extracardiac denervation. ${ }^{20}$ Accordingly, the destruction of GP rendered $96 \%$ of patients noninducible to $\mathrm{AF}^{21}$ Chiou and Zipes ${ }^{8}$ obtained selective vagal denervation of the atria, sinus, and AV nodes, with preserved ventricular innervation, with RF ablation of the atrial fat pad in a dog model. Moreover, Pappone and colleagues ${ }^{22}$ suggested that patients undergoing AF catheter-based RF ablation with "vagal" response, who demonstrated higher freedom from AF during follow-up, had GP destruction with loss of the GP function during the interventional procedure. Chen and coworkers ${ }^{23}$ demonstrated that a disturbed atrial electrophysiology mediated by higher vagal reflexes could play an 
important role in the genesis of paroxysmal $\mathrm{AF}$ and supraventricular tachycardias. Finally, Mehall and colleagues ${ }^{9}$ recently reported that autonomic GP are clustered around the interatrial groove and the ligament of Marshall, and that cardiac response to GP stimulation can be eliminated with surgical bipolar RF isolation, suggesting the potential for an increased freedom from AF with the addition of GP isolation to bilateral pulmonary vein isolation. ${ }^{9}$ However, there is no general agreement on the efficacy on postoperative AF of strategies targeted on autonomic nervous system: Alex and Guvendik $^{10}$ demonstrated that ventral cardiac denervation was not effective in reducing AF recurrences, whereas Jideus and colleagues ${ }^{11}$ showed thoracic epidural anesthesia to have no effects on postoperative AF. However, the resulting dominating vagotonic status induced by epidural anesthesia, reported by Jideus and colleagues, can explain the results of their study. We reported our experience with the combination of GP isolation to traditional RF mini-Maze procedure, and, as expected, we found a higher, but not significant, freedom from $\mathrm{AF}$ in those patients who underwent combined miniMaze plus GP isolation.

It is well known that the topography of cardiac ganglia in the adult human heart is not common basic or clinical knowledge, due in part to limited or inconsistent reports. ${ }^{24}$ Moreover, contradictory results are reported in the literature: Singh and colleagues ${ }^{24}$ showed 2 large populations of cardiac ganglia, the first lateral to the right pulmonary veins and inferior to the superior vena cava, near the sinoatrial node, and the second at the interatrial groove and coronary sulcus junction, near the AV node, but only small populations lateral to the left pulmonary veins. ${ }^{24}$ On the other hand, Leonhardt ${ }^{25}$ reported similar cardiac ganglia densities near the sinoatrial node and on the superior left atrial surface, also lateral to the right pulmonary veins. When pathologic-clinical correlations were investigated, Mehall and colleagues ${ }^{9}$ reported active GP preferentially located in the upper right and left fat pads along the pulmonary veins, with bilateral activity in the majority of patients. ${ }^{9}$ As did Leonhardt, ${ }^{25}$ we found GP clustered along the left pulmonary veins, although according to Singh and coworkers, ${ }^{24}$ the highest number of GP can be demonstrated on the fat pad of Waterston's groove, either on electrophysiologic stimulation or histologic analysis. Accordingly, our data agree with that of Mehall and colleagues, ${ }^{9}$ showing bilateral activity in the majority of patients, followed by unilateral right-sided GP activity. Moreover, on electrophysiologic study, we found active GPs to correlate with a higher number of clustered GPs detectable at histology. However, we did not found AF recurrences to correlate with a specific histologic pattern.

In-hospital results were significantly better in patients undergoing GP ablation because of the lower AF persistence at aortic declamping or hospital discharge. Our results suggested that GP function may affect the results of the AF ablation in the postoperative course, confirming the findings of Mehall and coworkers, ${ }^{9}$ who suggested that a persistent activity of unisolated active GP may explain the $10 \%$ to $40 \%$ recurrence rate of $\mathrm{AF}$ after the Maze procedure alone. Moreover, the impact of GP isolation in early postoperative SR recovery can be attributed to the elimination of the vagal reflexes and atrial susceptibility to AF, secondary to different "fibrillogenic" stimuli commonly encountered in the early postoperative phase, such as electrolytic imbalance, systemic inflammation, and hypovolemia. ${ }^{26}$

Fluid retention was one of the earliest recognized complications of the Maze procedure, related to biatrial appendectomy, lower atrial natriuretic peptide secretion, increased plasma arginine vasopressin and aldosterone levels, and temporary lack of response of the atrial baroreceptors. ${ }^{27} \mathrm{Ad}$ and colleagues $^{28}$ suggested that the abrupt cardioversion to SR decreases postoperative atrial natriuretic peptide levels, thus determining fluid retention. Despite the fact that biatrial appendectomy was performed in both groups, we found a lower need for furosemide at discharge and during follow-up in patients undergoing GP isolation. It can be hypothesized that the lower AF recurrences during hospitalization and the reduced AF, AFL, AT recurrences during followup after GP isolation, with a resulting better hemodynamic status, may explain the lower need for furosemide in this group. Accordingly, we found patients maintaining AF during follow-up to require more furosemide than patients converted to SR. Moreover, the reduced incidence of AF, AFL, and AT with and without antiarrhythmic therapy may favor the recovery of atrial function, thus resulting in a higher or earlier recovery of $\mathrm{E} / \mathrm{A}$ ratio (as found in group $\mathrm{B}$ ) at echocardiographic follow-up. However, more studies exploring hormonal response and correlations between electrophysiology and echocardiography are needed to substantiate such hypotheses.

Finally, it can be argued that a more aggressive approach with atrial denervation may favor the development of postoperative junctional rhythm or impair the heart rate autonomic control and reflexes. ${ }^{7,22}$ When junctional rhythm was considered, no differences were recorded in its prevalence in the 2 groups either at aortic declamping or during hospitalization, so no differences were recorded between the 2 groups in postoperative need for definitive pacemaker implantation. When autonomic imbalance was considered, it is well known from literature studies $^{7,22}$ that RF AF ablation modifies heart rate variability and reduces baroreflex sensitivity in the early postoperative course. ${ }^{22}$ However, we did not study heart rate variability; therefore, further studies are welcome to clarify this topic.

When echocardiographic findings are considered, previous literature studies have demonstrated that restoration of the SR is consistent with a reverse remodeling of the atrial diameters, volumes, and function. ${ }^{29,30}$ However, if the entity of such remodeling is due to the restoration of the rhythm or to the restoration of the underlying valve disease, the evaluation is still challenging. We confirmed our previous findings ${ }^{12}$ 
that patients undergoing mitral valve surgery demonstrated a significant reverse remodeling of atrial and ventricular diameters only in case of SR restoration, which correlates with a higher prevalence of E/A recovery at follow-up. Therefore, patients with persistence of AF do not undergo a reverse atrial remodeling or ventricular reverse remodeling, despite the relief of the underlying valve pathology, which may only ameliorate echocardiographic indexed left ventricular mass. Therefore, ventricular remodeling is demonstrated during follow-up only when SR maintenance is guaranteed. ${ }^{12}$ These findings correlated with the evidence that despite a comparable early postsurgical recovery of NYHA class in the 2 treatment groups, patients demonstrating AF persistence showed a lesser degree of symptomatic improvement during follow-up. ${ }^{12}$ However, no differences can be detected in atrial and ventricular remodeling between the 2 surgical treatments, demonstrating that GP isolation did not further affect reverse remodeling of the heart chambers. Finally, given the comparable atrioventricular remodeling and the absence of significant differences in follow-up freedom from AFL, it is not surprising that patients undergoing GP isolation + mini-Maze have similar follow-up freedom from acute heart decompensation and a comparable freedom from hospital readmission when compared with patients undergoing the left + right mini-Maze alone.

Extended GP isolation during AF ablation in mitral valve surgery improves SR recovery during hospitalization and adds a beneficial effect on follow-up freedom from $\mathrm{AF}$ and the recovery of atrial function during echocardiographic follow-up.

\section{Study Limitations}

The main limitation of the study is the limited number of patients enrolled in different periods; the lack of randomization to treatment is another limitation. Moreover, the type and duration of $\mathrm{AF}$ were heterogeneous. All of these limitations stem from the single-center design of the study, which, on the other hand, guaranteed uniformity of the perioperative management of the patient population throughout the experimentation. Certainly, further randomized studies or prolonged followup would be required to confirm the beneficial role of GP isolation during AF surgery.

The strengths of our study include its prospective design and the sequential echocardiographic assessment of both atrial and ventricular size and function before and after surgery. Furthermore, there are few literature data with which to compare the isolated Maze procedure with the Maze procedure plus GP isolation.

\section{References}

1. Cox JL, Schuessler RB, D'Agostino HJ, Stone CM, Chang BC, Cain ME, et al. The surgical treatment of atrial fibrillation. Development of a definitive surgical procedure. J Thorac Cardiovasc Surg. 1991;101: $569-83$.
2. Gillinov AM, Saltman AE. Ablation of atrial fibrillation with concomitant cardiac surgery. Semin Thorac Cardiovasc Surg. 2007;19:25-32.

3. Damiano RJ Jr, Schuessler RB, Voeller RK. Surgical treatment of atrial fibrillation: a look into the future. Semin Thorac Cardiovasc Surg. 2007; 19:39-45.

4. Haissaguerre M, Jais P, Shah DC, Takahashi A, Hocini M, Quiniou G, et al. Spontaneous initiation of atrial fibrillation by ectopic beats originating in the pulmonary veins. N Engl J Med. 1998;339:659-66.

5. Bettoni M, Zimermann M. Autonomic tone variations before the onset of paroxysmal atrial fibrillation. Circulation. 2002;105:2753.

6. Scherlag BJ, Nakagawa H, Jackman WM, Yamanashi WS, Patterson E, Po S, et al. Electrical stimulation to identify neural elements on the heart: their role in atrial fibrillation. J Intervent Card Electrophysiol. 2005;13: $1-6$.

7. Scanavacca M, Pisani CF, Hachul D, Lara S, Hardy C, Darrieux F, et al. Selective atrial vagal denervation guided by evoked vagal reflex to treat patients with paroxysmal atrial fibrillation. Circulation. 2006;114: 876-85.

8. Chiou CW, Zipes DP. Selective vagal denervation of the atria eliminates heart rate variability and baroreflex sensitivity while preserving ventricular innervation. Circulation. 1998;98:360-8.

9. Mehall JR, Kohut RM, Schneeberger EW, Taketani T, Merrill WH, Wolf RK. Intraoperative epicardial electrophysiologic mapping and isolation of autonomic ganglionic plexi. Ann Thorac Surg. 2007;83:538-41.

10. Alex J, Guvendik L. Evaluation of ventral cardiac denervation as a prophylaxis against atrial fibrillation after coronary artery bypass grafting. Ann Thorac Surg. 2005;79:517-20.

11. Jideus L, Joachimsson PO, Stridsberg M, Ericson M, Tyden H, Nilsson L, et al. Thoracic epidural anesthesia does not influence the occurrence of postoperative sustained atrial fibrillation. Ann Thorac Surg. 2001;72:65-71.

12. Onorati F, Esposito A, Messina G, di Virgilio A, Renzulli A. Right isthmus ablation reduces supraventricular arrhythmias after surgery for chronic atrial fibrillation. Ann Thorac Surg. 2008;85:39-49.

13. Calkins H, Cappato R, Crijns HJG, Damiano RJ Jr, Haissaguerre M, Jackman WM, et al. HRS/EHRA/ECAS Expert consensus statement on catheter and surgical ablation of atrial fibrillation: recommendations for personnel, policy, procedures and follow-up. Europace. 2007;9: 335-79.

14. Prasad SM, Maniar HS, Camillo CJ, Schuessler RB, Boineau JP, Sundt TM 3rd, et al. The Cox maze III procedure for atrial fibrillation: long-term efficacy in patients undergoing lone versus concomitant procedures. J Thorac Cardiovasc Surg. 2003;126:1822-88.

15. Gillinov AM. Ablation of atrial fibrillation in mitral valve surgery. Curr Opin Cardiol. 2005;20:107-14.

16. Gillinov AM, Blackstone EH, McCarthy PM. Atrial fibrillation: current surgical options and their assessment. Ann Thorac Surg. 2002;74: 2210-7.

17. Damiano RJ Jr. Alternative energy sources for atrial ablation. Judging the new technology. Ann Thorac Surg. 2003;75:329-30.

18. Sie HT, Beukema WP, Elvan A, Ramdat Misier AR. Long-term results of irrigated radiofrequency modified maze procedure in 200 patients with concomitant cardiac surgery. six years experience. Ann Thorac Surg. 2004;77:512-7.

19. Chiou CM, Chen SA, Kung MH, Chang MS, Prystowsky EN. Effects of continuous enhanced vagal tone on dual atrioventricular node and accessory pathways. Circulation. 2003;107:2583-8.

20. Ardell JL, Butler CK, Smith FM, Hopkins DA, Armour JA. Activity on in vivo atrial and ventricular neurons in chronically decentralized hearts. Am J Physiol. 1991;260:713-21.

21. Platt M, Mandapati R, Scherlag BJ. Limiting the number and extent of radiofrequency applications to terminate atrial fibrillation and subsequently prevent its inducibility. Heart Rhythm. 2004;(Suppl 1): S10-2.

22. Pappone C, Santinelli V, Manguso F, Vicedomini G, Gugliotta F, Augello G, et al. Pulmonary vein denervation enhances long-term benefit after circumferential ablation for paroxysmal atrial fibrillation. Circulation. 2004;109:327-34.

23. Chen YJ, Chen SA, Tai CT, Wen ZC, Feng AN, Ding YA, et al. Role of atrial electrophysiology and autonomic nervous system in patients with 
supraventricular tachycardia and paroxysmal atrial fibrillation. $J A m$ Coll Cardiol. 1998;32:732-8.

24. Singh S, Johnson PI, Lee RE, Orfei E, Lonchyna VA, Sullivan HJ, et al. Topography of cardiac ganglia in the adult human heart. $J$ Thorac Cardiovasc Surg. 1996;112:943-53.

25. Leonhardt H. Internal Organs. Autonomic nervous system; 3rd ed. Stuttgart: Georg Thieme, Verlag Ed; 1986:18-9.

26. Van Wagoner DR. Recent insights into the pathophysiology of atrial fibrillation. Semin Thorac Cardiovasc Surg. 2007;19:9-15.

27. Ad N, Tian YY, Verbalis J, Inahara SD, Cox JL. The effect of the maze procedure on the secretion of arginine-vasopressin and aldosterone. J Thorac Cardiovasc Surg. 2003;126:1095-100.
28. Ad N, Suyderhoud JP, Kim YD, Makary MA, De Groot KW, Lue HC, et al. Benefits of prophylactic continuous infusion of furosemide after the maze procedure for atrial fibrillation. $J$ Thorac Cardiovasc Surg. 2002;123:232-6.

29. Mattioli AV, Bonatti S, Bonetti L, Borella P, Mattioli G. Left atrial size and function after spontaneous cardioversion of atrial fibrillation and their relation to n-terminal atrial natriuretic peptide. Am J Cardiol. 2003;91:1478-81.

30. Fayad G, Le Tourneau T, Modine T, Azzaoui R, Ennezat PV, Decoene $\mathrm{C}$, et al. Endocardial radiofrequency ablation during mitral valve surgery: effect on cardiac rhythm, atrial size, and function. Ann Thorac Surg. 2005;79:1505-11. 